\title{
Emission line profiles in RY Scuti: a massive binary in a pre-WR stage?*
}

\author{
D. de Martino'i A.A. Vittone ${ }^{2}$, C. Rossi ${ }^{3}$, F. Giovannelli ${ }^{4}$ \\ 1 IUE Observatory, ESA-Vilspa \\ ${ }^{2}$ Osservatorio Astronomico di Capodimonte, Napoli \\ ${ }^{3}$ Istituto Astronomico dell'Università, Roma \\ ${ }^{4}$ Istituto di Astrofisica Spaziale, C.N.R., Frascati
}

\begin{abstract}
RY Scuti (HD 169515) is a massive $9^{\text {th }}$ mag eclipsing binary $\left(P_{\text {orb }}=\right.$ $\left.11.12^{d}\right)$ surrounded by a peculiar nebula. High resolution spectroscopic observations in $H \alpha$, He I $(\lambda 5876),[\mathrm{N} \mathrm{II}](\lambda \lambda 6548,6584),[\mathrm{A} \mathrm{III]}(\lambda 7136)$ and [S III] $(\lambda \lambda 9069,9532)$ are presented. All emission lines show a complex profile with two main structures: a sharp and strong red component and a very broad fainter complex blue one in which at least three sub-components can be detected. We have analysed these profiles in order to derive information on the velocity field in the nebula. Permitted and forbidden lines show the same velocity field indicating a common line forming region. The presence of a multi-structured blue component in all profiles gives evidence that velocity gradients are present within the nebula. Systemic corrected expansion velocities of +30 and $-45,-30,-9$ $\mathrm{Km} / \mathrm{s}$ are found for the red and the three blue components respectively. Weaker emission structures, reflecting the same asymmetries of the strong emissions, are observed close to the permitted lines at larger velocities $\left(V_{b}=-189 \mathrm{Km} / \mathrm{s}\right.$ and $\left.V_{r}=158 \mathrm{Km} / \mathrm{s}\right)$. The observed velocity field indicates an asymmetric mass outflow from the system very likely through the second Lagrange point rather than via stellar wind. The system should loose mass during the mass exchange phase. Using the masses $\left(M_{1}=39 M_{\odot}, M_{2}=49 M_{\odot}\right)$ and radii $\left(R_{1}=37 R_{\odot}, R_{2}=41 R_{\odot}\right)$ of the two components (Milano et al., 1981), and locating RY Scuti in the $[\log R, \log M]$ diagram for early type contact binaries from Leung and Schneider (1979), we find that this system has just evolved-off the Terminal Age Main Sequence. Moreover, placing RY Scuti in the $\left[\log P, \log M_{1} / M_{2}\right]$ diagram for $\mathrm{O}+\mathrm{O}$ and $\mathrm{WR}+\mathrm{OB}$ binaries of Massey (1981) we find that it is an evolutionary phase just preceeding a $\mathrm{WR}+\mathrm{OB}$ stage.
\end{abstract}

\section{REFERENCES.}

Leung, K.C. and Schneider, D.P.: 1979, IAU Symp. 83, eds. P.S. Conti and C.W.H. de Loore, p.265.

Massey, P.: 1981, Astrophys. J., 246, 153.

Milano, L., Vittone, A., Ciatti, F., Mammano, A., Margoni, R., Strazzulla, G.: 1981, Astron. Astrophys., 100, 59.

"Based on observations collected at ESO, La Silla, Chile

'On leave from Osservatorio Astronomico di Capodimonte, Naples 


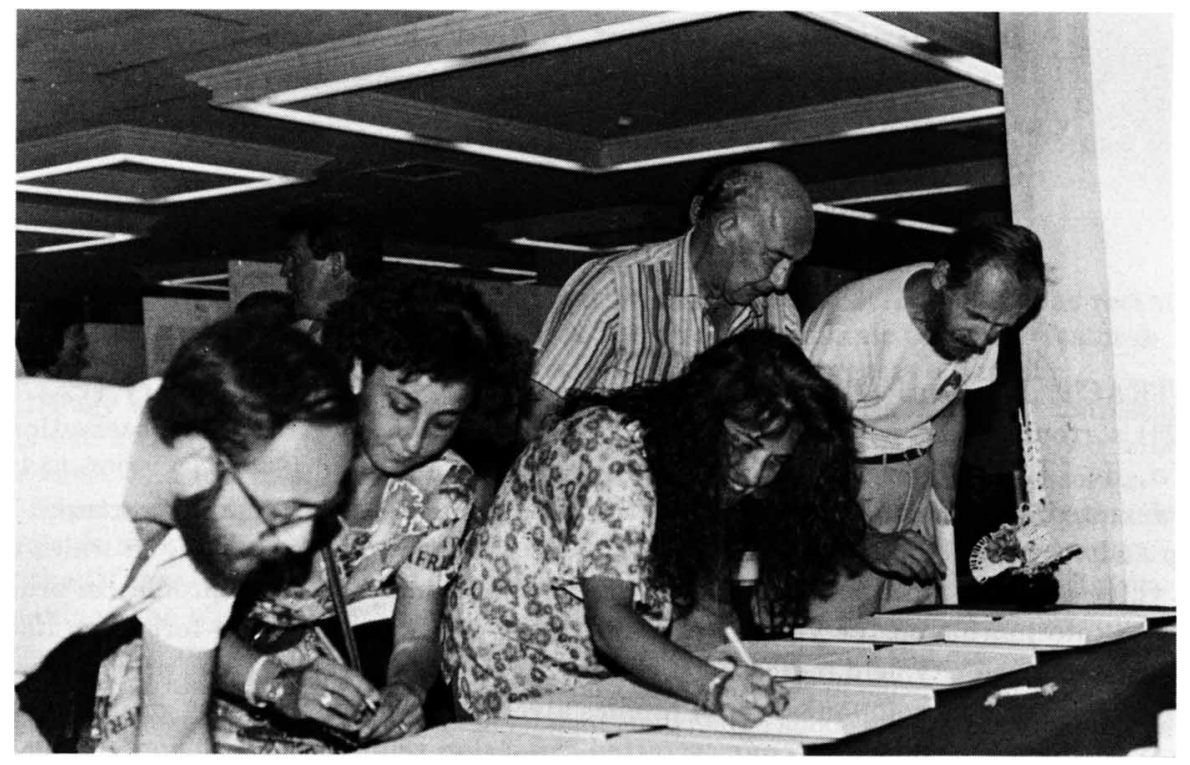

T'urolla, Cherepashchuk, de Martino, Matteucci, de Loore, de Groot 